\title{
Dissociated roles for the lateral and medial septum in elemental and contextual fear conditioning
}

\author{
Ludovic Calandreau, Robert Jaffard, and Aline Desmedt ${ }^{1}$ \\ Centre de Neurosciences Intégratives et Cognitives CNRS 5228, Université de Bordeaux I, 33405 Talence, France
}

\begin{abstract}
Extensive evidence indicates that the septum plays a predominant role in fear learning, yet the direction of this control is still a matter of debate. Increasing data suggest that the medial (MS) and lateral septum (LS) would be differentially required in fear conditioning depending on whether a discrete conditional stimulus (CS) predicts, or not, the occurrence of an aversive unconditional stimulus (US). Here, using a tone CS-US pairing (predictive discrete $\mathrm{CS}$, context in background) or unpairing (context in foreground) conditioning procedure, we show, in mice, that pretraining inactivation of the LS totally disrupted tone fear conditioning, which, otherwise, was spared by inactivation of the MS. Inactivating the LS also reduced foreground contextual fear conditioning, while sparing the higher level of conditioned freezing to the foreground (CS-US unpairing) than to the background context (CS-US pairing). In contrast, inactivation of the MS totally abolished this training-dependent level of contextual freezing. Interestingly, inactivation of the MS enhanced background contextual conditioning under the pairing condition, whereas it reduced foreground contextual conditioning under the unpairing condition. Hence, the present findings reveal a functional dissociation between the LS and the MS in Pavlovian fear conditioning depending on the predictive value of the discrete CS. While the requirement of the LS is crucial for the appropriate processing of the tone CS-US association, the MS is crucial for an appropriate processing of contextual cues as foreground or background information.
\end{abstract}

Extensive evidence has implicated the septum in fear and anxiety. On the one hand, previous studies have shown that damage to the septum could result in anxiolytic-like behavioral effects (Gray and McNaughton 1983, 2000). In this context, Gray (1982) proposed a model according to which activity of the septalhippocampal complex reflects a state of "anxiety" produced when there is a mismatch between predicted and actual sensory events. By preventing animals from detecting such a change, lesions or inactivation of the septum would then cause a decrease in fear or anxiety. On the other hand, septal lesions were also found to profoundly increase fear and anxiety-related behaviors (Thomas 1988), producing the "septal rage syndrome" in many species (Brady and Nauta 1953). This syndrome would result from a generalized disinhibition of fear leading to exacerbated defensive reactions. Hence, a long history of lesion studies indicates that the septum plays a predominant role in fear learning, yet the direction of this control is still a matter of debate.

The septal complex is anatomically heterogeneous. While the lateral septum (LS) receives a massive glutamatergic fiber input from the hippocampus by the fornix pathway, the medial septum (MS) sends impressive cholinergic and GABAergic projections to the hippocampus (Jakab and Leranth 1995; Swanson and Risold 2000).

Functionally, the MS has been shown to be critically involved in regulating some physiological properties of hippocampal neurons, such as theta rhythm, and place-related cells firing (King et al. 1998; Dragoi et al. 1999; Borhegyi et al. 2004). In line with this, electrolytic (Rawlins and Olton 1982; Kelsey and Vargas 1993), excitotoxic (Hagan et al. 1988), or also AMPA-induced lesions (McAlonan et al. 1995a,b) and more recently lesions with the selective cholinergic toxin 192IgG-saporin (Kirby and Rawlins 2003) demonstrated its involvement in both spatial and contextual fear conditioning. Regarding the LS, although several

${ }^{1}$ Corresponding author.

E-mail a.desmedt@cnic.u-bordeaux1.fr; fax 33-5-40008743.

Article is online at http://www.learnmem.org/cgi/doi/10.1101//m.531407. studies pointed out its involvement in fear learning, current results are somewhat equivocal as to whether the LS has a positive or negative influence on fear learning, a disparity that may depend on the behavioral test used (Sheehan et al. 2004). In addition, most of these studies have used large lesions that damaged most of the MS and LS. Thus, in order to specify the role of these two septal subregions in fear learning, we have, in mice, (1) mapped c-Fos expression in the LS and MS after two types of fear conditioning known to differentially require the hippocampal system (Desmedt et al. 1998, 1999; Calandreau et al. 2006; Trifilieff et al. 2006), and then (2) compared the effects of reversible neural inactivation of either the MS or the LS on the acquisition of these two types of fear conditioning. In the first procedure, a discrete conditional stimulus (CS) is systematically paired with an aversive unconditional stimulus (US), whereas in the second procedure, the CS and the US are unpaired. Because these two procedures result in either a predominant discrete CS-US (context in background) or context-US association (context in foreground) (Desmedt et al. 1998, 1999; Calandreau et al. 2005, 2006), such experimental design enables us to directly assess the relative contribution of both the LS and the MS to elemental and contextual conditioning.

\section{Results}

\section{Histology}

Representative guide cannulae placement and infusion sites are shown in Figure 1. Only data from subjects that exhibited correctly placed infusion sites were used in the analysis. Among a total of 74 animals, eight mice were excluded from the analysis because of either dislocated guide cannulae placement relative to the targeted septal subregion or infusion sites that reached the lateral ventricle.

\section{Immunohistochemistry}

In a preliminary experiment, mice were submitted either to a CS-US pairing or unpairing procedure, then killed for examina- 
A

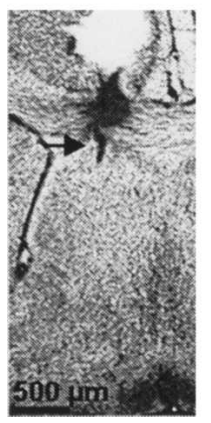

B

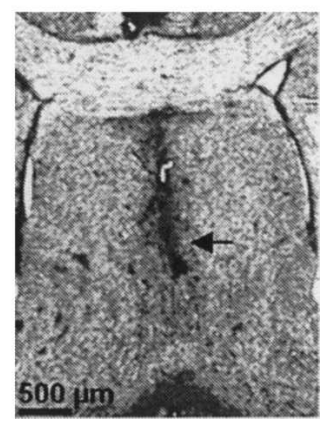

Figure 1. Representative guide cannulae placement and infusion sites (arrows) into the dorsal LS $(A)$ and MS $(B)$.

tion of c-Fos within the septum. Because c-Fos is a molecular marker commonly used to delineate learning-dependent circuits, we have mapped the patterns of c-Fos expression in the LS and MS after training in order to point out a differential involvement of these two subregions in fear conditioning as a function of the procedure used (i.e., CS-US pairing vs. unpairing).

As shown in Figure 2, levels of c-Fos expression were dependent on both the septal subregion analyzed and the conditioning procedure used. A two-way ANOVA with conditioning procedure (CS-US pairing vs. unpairing vs. naive) and septal subregion (LSd vs. LSv vs. MS) as between-groups factors indicated a significant subregion by group interaction $\left(F_{(4,60)}=23.494, P<0.001\right)$.

First, within the LS (Fig. 2A), c-Fos expression was different among the three groups in both the ventral LS (group effect: $\left.F_{(2,20)}=29.96, P<0.0001\right)$ and dorsal LS $\left(F_{(2,20)}=6.60\right.$, $P=0.006)$. However, post hoc analysis revealed that while both conditioning procedures resulted in similar increased c-Fos levels as compared to the naive condition in the ventral LS (both $P<0.01$ ), only the CS-US pairing procedure was found to produce significantly higher c-Fos levels with respect to the naive condition in the dorsal LS $(P<0.01)$.

Second, in the MS (Fig. 2B), an opposite pattern of c-Fos expression was observed (group effect: $F_{(2,20)}=10.54$, $P=0.0007)$. Specifically, post hoc analysis revealed that only the CS-US unpairing procedure produced significantly higher c-Fos levels with respect to the naive condition $(P<0.01)$.

The apparent opposite pattern of c-Fos expression revealed by the post hoc analyses was actually confirmed by a two-way ANOVA restricted to the critical modalities of the factors involved (CS-US pairing vs. unpairing) and (LSd vs. MS), which indicated a significant subregion by conditioning interaction $\left(F_{(1,28)}=4.49, P=0.043\right)$.

\section{Auditory-cue conditioning}

\section{Effects of LS inactivation}

Overall conditioned freezing to the tone CS (second vs. first block) was specific to the pairing (vs. unpairing) conditioning procedure and treatment (saline vs. lidocaine) (block $\times$ conditioning $\times$ treatment interaction: $\left.F_{(1,29)}=13.21, P=0.001\right)$ (Fig. 3A). Although there was an overall significant tendency for lidocaine-infused mice to display decreased levels of freezing as compared to saline-infused mice (effect of treatment: $\left.F_{(1,29)}=6.43, P=0.016\right)$, one can note that this effect was more marked in the pairing $\left(F_{(1,15)}=7.87, P=0.013\right)$ than in the unpairing condition $\left(F_{(1,14)}=0.33, \mathrm{~ns}\right)$. This is mainly because the training-dependent increase in freezing due to the tone presentation was observed in saline-infused mice (block $\times$ con- ditioning interaction: $\left.F_{(1,12)}=32.02, P=0.0001\right)$ but was abolished by inactivation of the LS (block $\times$ conditioning: $F_{(1,17)}=1.24$, ns). These results demonstrate that the LS is required for the acquisition of auditory-cue fear conditioning.

\section{Effects of MS inactivation}

Lidocaine-infused mice again displayed overall decreased levels of freezing as compared to saline-infused mice (effect of treatment: $\left.F_{(1,29)}=5.86, P=0.021\right)$. Nevertheless, the training-dependent increase in freezing observed during the second block (as compared to the first one; block $\times$ conditioning: $F_{(1,29)}=11.63$, $P=0.001)$ was not dependent on the treatment condition (block $\times$ conditioning $\times$ treatment interaction: $F_{(1,29)}=0.002$, ns). Compared to unpaired mice, both saline-infused mice and lidocaine-infused mice trained under the pairing condition displayed a significant increase in freezing during the tone presentation (block $\times$ conditioning interaction in saline-infused mice: $F_{(1,15)}=4.86, P=0.04$; block $\times$ conditioning interaction in lidocaine-infused mice: $\left.F_{(1,14)}=7.72, P=0.014\right)$. These results demonstrate that the MS is not required for the acquisition of auditory-cue conditioning.

It must be noticed that statistical analyses restricted to the pre-tone block (i.e., first block) revealed that overall conditioned freezing during this period was not specific to the pairing (vs. unpairing) procedure or treatment (saline vs. lidocaine) whatever the targeted septal area (LS: treatment $\times$ conditioning interaction: $F_{(1,29)}=0.078, \mathrm{~ns}$; MS: treatment $\times$ conditioning interaction: $\left.F_{(1,29)}=0.96, \mathrm{~ns}\right)$.
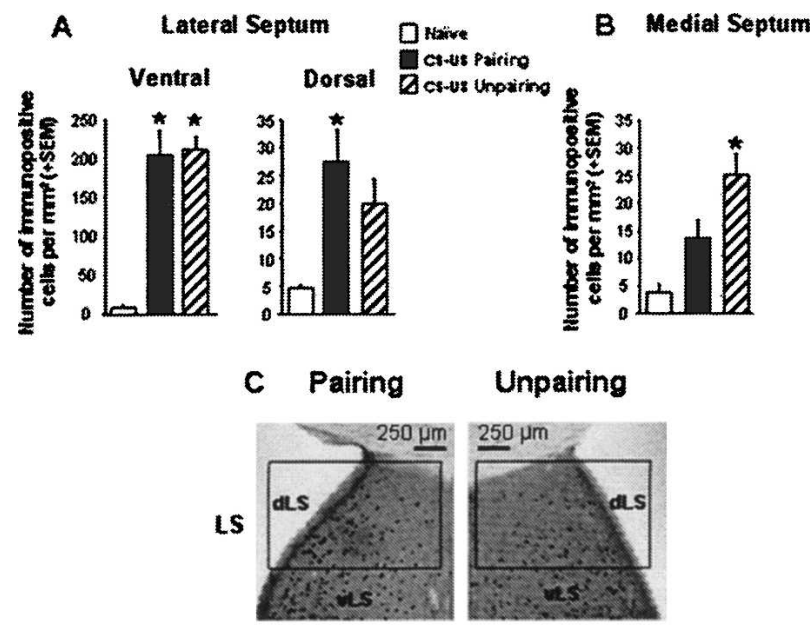

Unpairing
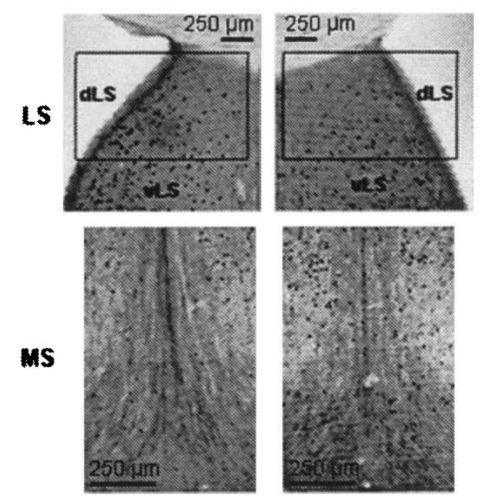

Figure 2. Number of c-Fos-immunopositive cells per square millimeter (mean + SEM) in the ventral LS $(A$, left), the dorsal LS $(A$, right), and the MS $(B)$ in mice submitted to either a CS-US pairing $(n=8)$ or unpairing $(n=8)$ conditioning procedure and in naive animals $(n=7)$. (C) Representative photomicrographs depicting c-Fos expression within the dorsal, ventral LS (dLS, vLS) and MS in mice submitted to the CS-US pairing or unpairing procedure. $\left({ }^{*}\right)$ Significantly different from the naive group, $P<0.05$. 

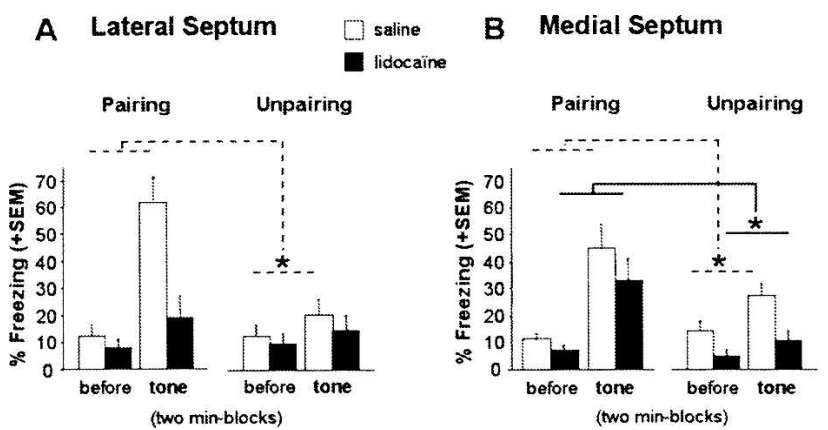

Figure 3. Auditory-cue test. Mean percentage freezing (+SEM) on successive 2-min blocks before (first block) and during (second block) tone presentation in animals that received lidocaine $(n=35)$, or $\mathrm{NaCl}(n=31)$ infusions into the $\mathrm{LS}(A)$ or into the MS $(B)$ before acquisition with either a CS-US pairing or unpairing procedure. $\left(^{*}\right)$ Statistically significant block $\times$ conditioning interaction, $P<0.05$.

\section{Contextual conditioning}

\section{Effects of $L S$ inactivation}

The effects of LS inactivation on contextual freezing tended to be dependent on the conditioning procedure used (conditioning $\times$ treatment: $\left.F_{(1,29)}=3.98, P=0.055\right)$. This close to significance interaction was strengthened by the fact that lidocaine infusions into the LS resulted in a significantly diminished conditioned freezing to the context in mice trained with the unpairing procedure (effect of treatment: $F_{(1,14)}=5.21, P=0.038$ ) (Fig. 4A), while leaving unchanged contextual freezing in mice trained with the pairing procedure $\left(F_{(1,15)}=0.048\right.$, ns). Nevertheless, both saline- and lidocaine-infused mice displayed significantly more conditioned freezing to the context when trained with the unpairing (context in the foreground) than with the pairing procedure (context in the background; effect of conditioning in SAL: $F_{(1,12)}=54.40, P<0.0001$; in LIDO: $\left.F_{(1,17)}=10.68, P=0.004\right)$.

\section{Effects of MS inactivation}

Lidocaine infusions into the MS resulted in a significantly diminished conditioned freezing to the context in mice trained with the unpairing procedure (effect of treatment: $F_{(1,15)}=8.83$, $P=0.009$ ), while significantly increasing contextual freezing in mice trained with the pairing procedure (effect of treatment: $F_{(1,14)}=5.51, P=0.034$ ) (Fig. $\left.4 \mathrm{~B}\right)$. As a consequence, the typical training-dependent effect observed in saline-infused mice on contextual freezing was abolished in lidocaine-infused mice (conditioning $\times$ treatment interaction: $F_{(1,29)}=13.98$, $P=0.0008)$. Specifically, while saline-infused mice displayed more conditioned freezing to the context when trained with the unpairing than with the pairing procedure (effect of conditioning: $\left.F_{(1,15)}=16.61, P=0.001\right)$, lidocaine-infused mice displayed similar levels of freezing in both training conditions (no conditioning effect: $F_{(1,14)}=1.95$, ns).

\section{Discussion}

So far, the septum had been shown to be involved in fear conditioning (Gray and McNaughton 1983; Sparks and LeDoux 2000), yet previous studies had not provided direct evidence for distinct roles of the LS and MS in elemental and contextual fear conditioning. This is probably because most of the septal damage or inactivation extended to the whole septum and also because analysis was restricted to either cue or contextual conditioning. The present findings reveal a functional dissociation between the LS and the MS in Pavlovian fear conditioning. Inactivating the LS totally disrupted auditory fear conditioning, which, otherwise, was spared by inactivation of the MS. In addition, while reducing contextual fear conditioning under the unpairing condition, inactivating the LS spared the training-dependent level of contextual freezing (i.e., more freezing to the foreground than to the background context). In contrast, inactivation of the MS specifically abolished this training-dependent effect.

From a pharmacological point of view, several previous observations indicate that the active site of the injected volume of lidocaine was mostly limited either to the LS or the MS. First, previous technical (Peterson 1998) and functional (Malpeli and Schiller 1979) studies examining the diffusion of micro-injected drugs into discrete brain areas allowed us to estimate that a 0.15 $0.2-\mu \mathrm{L}$ volume administered over $3 \mathrm{~min}$ resulted in a sphere of functional inactivation of $\sim 200-400 \mu^{3}$ in the brain. This implies, in our experiment, that the drug spread was mostly limited to the targeted region. Second, Martin (1991) demonstrated, using autoradiography on brain slices, that $1 \mu \mathrm{L}$ of lidocaine spreads in a radial fashion to a distance of $1.7 \mathrm{~mm}$ from the site of injection in both cortical and subcortical tissues and that its peak of activity occurs within 10 min post-infusion. Furthermore, and in accordance with these findings, recent data demonstrated that a volume of $0.25 \mu \mathrm{L}$ of lidocaine (2\%) specifically inactivated either the dorsal CA1 or CA3 of the hippocampus (Daumas et al. 2005). Third, we recently demonstrated that a volume of $0.15 \mu \mathrm{L}(2 \%)$ specifically inactivated either the LA or the BLA nucleus of the amygdala (Calandreau et al. 2005). Finally, and more importantly, the observed dissociation of the behavioral effects of the injections depending on the targeted septal subregion indicates that there exists only minimal overlap, if, indeed, there was any overlap at all between the two spheres (chosen volume of the bolus $0.20 \mu \mathrm{L}, 2 \%$ ) of drug diffusion.

\section{Dissociated roles of the LS and MS in auditory-cue conditioning}

The present study provides direct evidence for a different contribution of the LS and MS to the acquisition of auditory-cue conditioning. LS inactivation totally abolished the specific increase in conditioned freezing to the simple tone CS in mice trained with the CS-US pairing procedure. In contrast, MS inactivation, while diminishing levels of freezing during the auditory-cue test, spared the acquisition of auditory-cue conditioning. The overall diminished level of freezing after both MS and LS inactivation is generally consistent with previous findings reporting anxiolyticlike effects of septal lesions (Gray and Mc Naughton 1983; Pesold and Treit 1992; Menard and Treit 1996). However, past studies on the partial reinforcement extinction effect reported dissociated
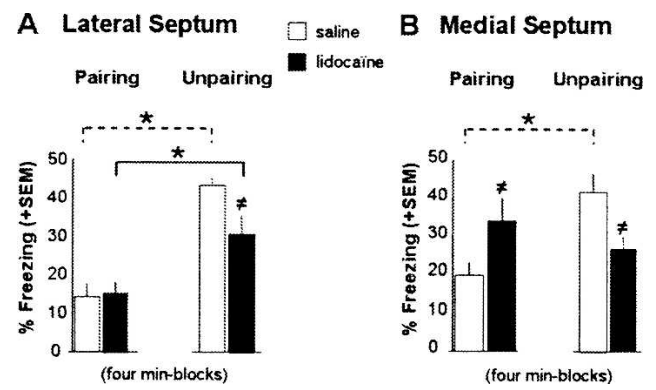

Figure 4. Context test. Mean percentage freezing (+SEM) on 4-min block during re-exposure to the conditioning context in animals that received lidocaine or $\mathrm{NaCl}$ infusions into the LS $(A)$ or the MS $(B)$ before acquisition with either a CS-US pairing or unpairing procedure. (*) Statistically significant conditioning effect (CS-US pairing vs. unpairing), $P<0.05 ;(\neq)$ treatment effect (LIDO vs. $\mathrm{NaCl}), P<0.05$. 
roles of the MS and LS depending on the stressful situation. In particular, while LS lesions decreased resistance to extinction (in a partial reinforcement condition, nonpredictable US), MS lesions tended to increase resistance to extinction (in a continuous reinforcement condition, predictable US) (Feldon and Gray 1979). In the present study, the decreases in levels of freezing were also dependent on both the training procedure and the targeted septal subregion. More specifically, and similar to Feldon's observations, LS inactivation actually decreased contextual fear conditioning (in the unpairing condition, nonpredictable US), while MS inactivation increased contextual fear conditioning (in the pairing condition, predictable US). Even if a direct comparison of the effects observed in the Feldon's studies and the present one must be taken with caution because of several technical and methodological differences, the similarities observed between these studies indicate a specific, although different, contribution of the LS and MS in fear conditioning as a function of the cognitive process engaged.

The presently observed disruption and preservation of significant tone fear conditioning after LS and MS inactivation, respectively, are congruent with previous studies that showed a functional segregation between lateral and medial septal cells. Using field potential recordings, we previously reported bidirectional changes in hippocampal-lateral septal synaptic excitability as a function of the predictive value of the tone CS for the occurrence of the US (Garcia and Jaffard 1996; Desmedt et al. 1998, 2003a). In line with this, cell unit recordings in the LS also revealed bidirectional changes in the cell firing rate depending on whether or not a CS signaled an aversive US. By comparison, most of medial septal cells are less sensitive to the CS-US contingency (Thomas and Yadin 1980; Yadin and Thomas 1981; Thomas et al. 2005), but interestingly, their predominant responses appear to be the reverse of what is observed in the LS (Thomas et al. 1991). In accordance with these studies, the present immunohistochemical data showed an opposite pattern of c-Fos expression in the dLS and MS depending on whether the discrete tone CS was predictive or not of the footshock US. While a significant increase in c-Fos expression was observed in the dLS, but not in the MS, after a CS-US pairing procedure (context in the background), an inverse pattern of results was observed after a CS-US unpairing procedure (context in the foreground). Altogether, these data support the idea of a crucial requirement of the LS in elemental fear conditioning, and suggest a main contribution of the MS to contextual conditioning (see also Desmedt et al. 2003b).

\section{Role of the LS in contextual conditioning}

Pre-training inactivation of the LS not only abolished auditorycue conditioning (pairing condition), but also reduced conditioned freezing to the foreground context (unpairing condition). Previous studies indicate that the absence of significant effect of LS inactivation on freezing to the tone in the unpairing condition and on contextual freezing in the pairing condition is unlikely to be due to a floor effect. First, using the same experimental protocol, we previously reported that pre-training intrahippocampal infusion of scopolamine (Calandreau et al. 2006) and inactivation of the basolateral nucleus of the amygdala (Calandreau et al. 2005) actually diminished the levels of contextual freezing even in the pairing condition. This indicates that even if control animals trained under the pairing procedure display a lower level of contextual freezing than those trained under the unpairing procedure, it is still possible to reduce such a low level of freezing. Consequently, the sole floor effect argument cannot explain why inactivation of the dorsal LS has no significant effect on contextual freezing in the pairing condition in the present study. Second, our results demonstrate that lidocaine infusion into the dorsal LS does not prevent mice from appropriately (depending on the CS-US contingency) processing contextual cues as foreground or background information. Indeed, lidocaineinfused mice trained under the unpairing condition still display more conditioned freezing to the context than those trained under the pairing condition. This indicates that lidocaine has no effect on conditioned freezing per se. Indeed, if it were the case, no difference in freezing behavior should be observed between the two training conditions. This also indicates that inactivation of the LS does not prevent mice from detecting the different CS-US contingency training rules. Otherwise, mice could not, as a function of such a rule, appropriately process the context as background or foreground information.

Consequently, the main explanation for the presently observed effect of inactivation of the dorsal LS is that it prevents mice from "amplifying" the processing of the main predictor (either the tone or the context depending on the CS-US contingency) in the training environment. Given the pivotal anatomical position of the LS within the limbic system, this trainingdependent effect of LS inactivation might reflect alteration in the encoding neural system of reinforcing stimuli. In particular, lesion and electrophysiological studies revealed functional interactions between the LS and the amygdala (Melia et al. 1992; Desmedt et al. 1998; see also Desmedt et al. 1999). Since the amygdala has been shown to be differentially required for fear conditioning as a function of the procedure used (Calandreau et al. 2005), it is likely that the LS may also be differentially required for a preferential tone (CS-US pairing) or contextual fear conditioning (CS-US unpairing).

Nevertheless, while decreasing contextual freezing in mice trained with the unpairing procedure, yet inactivation of the LS spared a differential freezing level to the context depending on the CS-US contingency. Indeed, as observed in saline-infused mice, lidocaine-infused mice exhibited higher freezing levels to the foreground than to the background context. This trainingdependent effect on contextual fear conditioning can be explained by the cue validity effect. Associative learning theories such as that of Rescorla and Wagner (1972) can readily explain this result. Briefly, the Rescorla-Wagner model states that a reinforcer can sustain only a limited amount of associative strength; thus, simultaneously presented cues are supposed to compete with one another as the best predictor of the US occurrence. In the pairing condition, the tone is consistently followed by the US, whereas the context is continuously present during training. Consequently, the tone becomes a strong predictor to the detriment of the context and captures most of the fixed amount of the associative strength sustained by the US. In the unpairing condition, the tone is never followed by the US, while the context is the sole stimulus "directly" associated with the occurrence of the US. In that condition, the context is a better predictor than the tone for the US occurrence and captures most of the associative strength. Interestingly, this training-dependent effect on contextual freezing was specifically abolished by MS inactivation, which reduced contextual freezing under the unpairing condition and, conversely, potentiated contextual freezing under the pairing condition.

\section{MS and adaptive processing of contextual cues}

The present impairment of foreground contextual conditioning is in agreement with previous studies showing a deficit in hippocampal-dependent learning following MS lesions (Rawlins and Olton 1982; Hagan et al. 1988; Kelsey and Vargas 1993). Because of the strong anatomical and functional interlink between the MS and the hippocampus, MS lesions are expected to produce the 
same disrupting effect as hippocampal lesions on spatial learning and contextual fear conditioning (Selden et al. 1991; Phillips and LeDoux 1992; Richmond et al. 1999). It is important to note, however, that Bannerman et al. (2004) showed that MS lesions had no effect on conditioned contextual freezing. This absence of deficit is reminiscent of the lack of impairment of contextual conditioning observed in some studies after hippocampal lesions (Maren et al. 1997; Frankland et al. 1998; Gerlai 1998). Evidence strongly suggests that under certain conditioning procedures, which, for instance, do not use any discrete CS (i.e., shock alone condition), animals could associate the footshock US with a simple salient contextual cue (e.g., footshock grid). Such a procedure, actually used in the study by Bannerman et al. (2004), might, in fact, promote a contextual conditioning based on an elemental associative strategy, which would not require the integrity of the hippocampus (Maren et al. 1997; Frankland et al. 1998; Cho et al. 1999; Holland and Bouton 1999; Anagnostaras et al. 2001) or that of the MS. Moreover, in the Bannerman experiment, the absence of unpaired phasic tone CS could reduce the competition between cues to acquire associative strength. This conditioning situation with "low competition" would, thus, not critically require the MS. On the contrary, in the present study, the use of an unpaired phasic tone CS would favor a competition between a simple phasic contextual CS (i.e., the tone) and the polymodal static contextual cues. This competition would critically require the MS for the selection of the best predictor (the polymodal context). Consequently, contrary to what was observed in the Bannerman experiment (Bannerman et al. 2004), inactivation of the MS actually results in disrupting contextual fear conditioning in our training condition, providing thereby a critical role of the MS in the processing of the relative cue validity.

The potentiation of contextual freezing under the pairing condition is borne out by previous studies showing that septal lesions enhanced contextual fear conditioning when the delivery of shock is signaled (McAlonan et al. 1995b; Sparks and LeDoux 1995). While in accordance with these data, our present findings reveal that the MS actually contributes to the appropriate processing level of contextual information as a function of the predictive value of the discrete CS and strengthen the view of a critical role of the MS in the processing of the relative cue validity. Indeed, in contrast to the control condition, pre-training inactivation of the MS produced similar levels of contextual freezing after both types of conditioning procedures. In line with this, previous data indicated that the subcortical cholinergic projection to the hippocampus might increase the signal-to-noise ratio by decreasing noise (i.e., background contextual cues) (McAlonan et al. 1995b). More recently, we demonstrated that the level of the hippocampal cholinergic transmission is causally related to the processing of contextual stimuli as foreground or background information depending on the predictive value of the simple tone CS (Calandreau et al. 2006). Thus, inactivating the MS would result in altering the functioning of the septalhippocampal cholinergic neurotransmission and consequently would prevent animals from processing contextual cues as less predictive cues than the simple tone CS under the pairing condition. As a result, animals could not consign the contextual cues in the background in this training condition. Similarly, this inactivation would also prevent animals from optimally processing contextual cues in the foreground when trained with the unpairing procedure. Hence, these findings reveal that the MS is not only required for processing contextual cues but is mostly required for an adaptive, that is CS-US contingency-dependent, processing of the contextual cue validity.

In conclusion, the present study demonstrates dissociated roles for the LS and MS in elemental and contextual fear condi- tioning. While the LS is crucially required for the simple toneshock association, the MS is involved in the context-US association. More specifically, these findings show that both septal subregions are involved in the processing of the CS-US contingency. Nevertheless, while the LS has a key role in elemental conditioning, the MS specifically subserves the appropriate processing of contextual cues as foreground or background information. Although further experiments are needed to unveil the mechanisms and specific neural circuitry involved in this newly revealed functional dissociation, the present findings highlight the LS and MS contributions to adaptive conditioned emotional responses as a function of the learning situation considered.

\section{Materials and Methods}

\section{Subjects and surgery}

The experiments were conducted on naive adult (4-6 mo) male mice (C57Bl/6 JI Co, IFFA Credo) weighing 27-32 g. They were housed individually $7 \mathrm{~d}$ before the experiments and maintained in an animal room $\left(23^{\circ} \mathrm{C}\right)$ with a 12 -h artificial light/dark cycle and with ad libitum access to food and water. Light hours were between 07:00 and 19:00. All behavioral testing was carried out during the light phase of the cycle.

Mice were anesthetized with Avertin (tribromoethanol; 10 $\mathrm{mL} / \mathrm{kg}$, i.p.) and secured in a Kopf stereotaxic apparatus. The position of the incisor bar was adjusted, if necessary, so that the bregma and the lambda were in the same horizontal plane. After taking a reading of the dorsal surface of the sagittal sinus, a small piece of hemostatic sponge was used to avoid excessive bleeding. Stainless-steel guide cannulae (26 gauge, $8 \mathrm{~mm}$ length) were implanted either $1 \mathrm{~mm}$ above the MS (3.6 $\mathrm{mm}$ below the skull surface, $0.9 \mathrm{~mm}$ anterior to bregma, and $0 \mu \mathrm{m}$ lateral from midline), or simultaneously bilaterally $1 \mathrm{~mm}$ above the dorsal LS (2.1 $\mathrm{mm}$ below the skull surface, $0.9 \mathrm{~mm}$ anterior to bregma, and 0.4 $\mathrm{mm}$ lateral from midline) (Franklin and Paxinos 1997), then fixed in place with dental cement and two jewel screws attached to the skull. Mice were then allowed to recover in their home cages in the animal room for at least $8 \mathrm{~d}$ before behavioral experiments. The choice of the placement of the cannulae at the dorsal LS was based on previous experiments showing that the hippocampal-(dorsal) lateral septal glutamatergic neurotransmission was differentially involved in fear conditioning depending on whether the CS and US are paired or unpaired (Garcia and Jaffard 1996; Desmedt et al. 1998, 2003a).

\section{Intracerebral infusions}

For infusion, the dummy stylets that obturated the free end of the guide cannulae were removed, and 32-gauge stainless-steel cannulae $(9 \mathrm{~mm})$, attached to $1-\mu \mathrm{L}$ Hamilton syringes (PolyLabo) with polyethylene catheter tubing, were inserted through the guides. The syringes were fixed in a constant rate infusion pump (Roucaire), and 2\% lidocaine (2-diethylamino- $N-[2,6-$ dimethylphenyl]-acetamide hydrochloride; Sigma) was bilaterally infused $(0.2 \mu \mathrm{L}$ per side) into the LS or unilaterally infused $(0.2 \mu \mathrm{L})$ into the MS over a 3-min period during which the mouse was maintained in its home cage. Infusion flow rates were checked visually. The cannulae were left in place for a further 3 min before removal to allow diffusion of the drug away from the cannula tip (Desmedt et al. 1999; Calandreau et al. 2005, 2006).

The injection volume was chosen on the basis of previous findings reporting that this resulted in selective functional inactivation of $200-400-\mu \mathrm{m}^{3}$ brain regions (Malpeli and Schiller 1979), that is, in our experiment, a drug spread limited to the targeted region. Moreover, Tehovnick and Sommer (1997) reported that monkey cerebral cortex units were inactivated $<8$ min after intracortical lidocaine injection, and that they gradually recovered, regaining much of their initial activity within 30 min after the injection. By inducing a peak of inactivation within the targeted brain site lasting from 3 to $10 \mathrm{~min}$, this pre-training infusion was thought to induce a functional inactivation of either the LS or the MS that was limited mostly to the acquisition 
phase of conditioning. One control group was submitted to the same procedure except that animals received $\mathrm{NaCl}$ infusion.

\section{Behavioral procedure}

Classical fear conditioning took place in a Plexiglas chamber $(30 \times 24 \times 22 \mathrm{~cm}$ high $)$ given access to the different visualspatial cues in the experimental room. The floor of this conditioning chamber consisted of stainless steel rods $(2 \mathrm{~mm}$ diameter), spaced $5 \mathrm{~mm}$ apart and connected to a shock generator (Imetronic). The four sides of the chamber and the rods of the floor were cleaned with 70\% ethanol before each trial. For acquisition, half of the animals was given two explicit pairings of the tone CS $(63 \mathrm{~dB}, 1 \mathrm{kHz}, 15 \mathrm{sec})$ and a footshock US $(0.7 \mathrm{~mA}, 50 \mathrm{~Hz}$, $3 \mathrm{sec})$. Specifically, each animal was placed in the conditioning chamber for $4 \mathrm{~min} ; 100 \mathrm{sec}$ after being placed into the chamber, a tone was presented and was immediately followed by a footshock. Sixty seconds later, the same tone-shock pairing was presented. After an additional delay of $40 \mathrm{sec}$, each mouse was removed from the conditioning chamber and replaced in its home cage. In this training condition, the prevalent association is the elemental CS-US association, which consigns the conditioning context to the background. The other half of the subjects were trained under the unpairing tone-shock procedure, which differed from the previous training condition in that the two shocks and the two tones were pseudo-randomly distributed. Specifically, $100 \mathrm{sec}$ after being placed into the chamber, animals received a footshock, then after a 20 -sec delay, a tone was presented. Finally, after a 30-sec delay, the same tone and the same shock spaced out $30 \mathrm{sec}$ apart were presented, and each animal was replaced in its home cage after a final delay of $20 \mathrm{sec}$. This explicitly unpaired arrangement of tone and shock is known to enable the contextual cues to be processed as the primary stimuli to enter into association with the US (i.e., context in foreground) (Desmedt et al. 1998, 1999; Calandreau et al. 2005, 2006).

Twenty-four hours later, while animals were drug free, they were all tested for freezing behavior (measured second-bysecond), used as an index of conditioned fear and defined as a lack of all movement, except for respiratory-related movements (Fanselow 1980). First, all mice were submitted to the auditory cue test: they were maintained in their home cage, and two successive recording sessions of behavioral responses were performed: one prior to (first $2 \mathrm{~min}$ ) and one during (next $2 \mathrm{~min}$ ) the tone presentation. For the tone test, animals together with their home cage were placed in the conditioning room. It is important to note that the context was slightly modified for this test: the luminosity slightly increased (from 60 to 100 lux), and the context in which the home cage was placed was cleaned with acetic acid (1\%) instead of alcohol (70\%) before each testing trial. Finally, because the home cage is in opaque Plexiglas, it prevents mice from having a perceptual access to the surrounding visuospatial cues of the training environment.

Two hours later, all mice were submitted to the context test: they were re-exposed for $4 \mathrm{~min}$ to the conditioning chamber. Animals were continuously videotaped for off-line scoring of freezing. Freezing was evaluated manually and measured secondby-second. This behavioral procedure has been repeatedly used and fully described in previous experiments (Desmedt et al. 1998, 1999; Calandreau et al. 2005, 2006).

\section{Histology}

After completion of the behavioral study, animals were given an overdose of sodium thiopental $(120 \mathrm{mg} / \mathrm{kg})$ and transcardially perfused with physiological saline, followed by $10 \%$ buffered formalin. Brains were post-fixed in formalin-saccharose 30\% solution for $1 \mathrm{wk}$, frozen, cut coronally on a sliding microtome into $50-\mu \mathrm{m}$ sections that were mounted on a gelatin-coated slide, and stained with thionine in order to evaluate the cannulae placements.

\section{Immunohistochemistry}

Twenty-three mice were deeply anesthetized using avertin (10 $\mathrm{mL} / \mathrm{Kg}$, i.p.) $1.5 \mathrm{~h}$ after conditioning with either a CS-US pairing $(n=8)$ or unpairing $(n=8)$ procedure and perfused intracardiacally with $100 \mathrm{~mL}$ of $4 \%$ paraformaldehyde (PFA) in $0.1 \mathrm{M}$ $\mathrm{Na}_{2} \mathrm{HPO}_{4} / \mathrm{NaH}_{2} \mathrm{PO}_{4}$ buffer $(\mathrm{pH} 7.5)$ at $4^{\circ} \mathrm{C}$. Additional mice (NAIVE, $n=7$ ) were taken directly from their home cage in the animal room and sacrificed for immunohistochemistry analysis. Brains were removed, post-fixed overnight in the same fixative solution as described above, and then sectioned using a Vibratome (Leica). Free-floating sections (50 $\mu \mathrm{m}$ thin) were rinsed in phosphate buffer saline (PBS) and pretreated with $0.3 \% \mathrm{H}_{2} \mathrm{O}_{2}$ in PBS to reduce endogenous peroxidase activity. After four rinses (15 min each), sections were incubated in a blocking solution ( $2 \%$ bovine serum albumin $[\mathrm{BSA}] / 2 \%$ normal goat serum $/ 0.2 \%$ Triton $\mathrm{X}-100)$ for $1 \mathrm{~h}$ at room temperature. Then, they were incubated in primary polyclonal rabbit anti-c-Fos (Oncogene Research Products; 1:20,000 dilution) antibodies in the blocking solution overnight at room temperature. Subsequently, sections were washed with PBS and incubated for $2 \mathrm{~h}$ at room temperature with biotinylated goat anti-rabbit IgG (Jackson Immunoresearch; 1:2000 in PBS) followed by $2 \mathrm{~h}$ at room temperature in the avidin-biotin peroxidase complex (Vectastain Elite kit; Vector Laboratories). Sections were rinsed in PBS and then PB. The peroxidase reaction end-product was visualized by incubating sections in $0.05 \mathrm{M}$ Tris ( $\mathrm{pH} 7.6$ ) containing 3,3'-diaminobenzidine tetrahydrochloride (DAB, $0.037 \%$ ) as chromogen and hydrogen peroxide $(0.015 \%)$ for $15 \mathrm{~min}$. Finally, immunolabeled sections were washed in $\mathrm{PB}$, mounted on gelatin-coated slides, dehydrated, and coverslipped. A videocamera (SONY DXC-950P) attached to a microscope (OLYMPUS BX 50) and connected to an analysis software (Biocom; Visiolab 2000, V4.50) was used for labeled cell quantification. Immunochemistry cell counting was restricted to dorsal and ventral LS as well the medial nucleus of the medial septal complex. Six measurements (three per hemisphere) were made for dorsal and ventral LS and averaged. Three measurements were made for the MS and averaged. Quantifications were carried out at $10 \times$ magnification. Sections from the LS (dorsal and ventral part) and the MS were between bregma $1 \mathrm{~mm}$ and bregma $0.6 \mathrm{~mm}$ (Franklin and Paxinos 1997). The dorsal LS (LSd) is the region of the lateral septal area positioned immediately under the corpus callosum and close to the lateral ventricule. The ventral lateral septal area (LSv) corresponds to both the intermediate LS plus the ventral LS according to Franklin and Paxinos (1997). The choice of this subdivision was made on the basis of previous observations. Using electrophysiology, Garcia and Jaffard (1996) and also Desmedt et al. $(1998,2003 a)$ showed that the LSd was differentially involved in Pavlovian fear learning depending on whether a pairing or an unpairing tone CS-US procedure was used. On these bases, it was expected that levels of c-Fos would be different in this region of interest (but not necessarily in the LSi and LSv) as a function of the training procedure used. Consequently, c-Fos cell counting in the dorsal LS was dissociated from the c-Fos cell counting in the intermediate LS and ventral LS, which were not performed separately. Regarding the medial septum (MS), cell counting was restricted to the medial nucleus of the medial septal complex according to Franklin and Paxinos (1997). This choice was based on previous data demonstrating that the medial nucleus constitutes the main (cholinergic) input to the hippocampus.

c-Fos-positive cells were automatically detected by the computer in rectangular boxes of $100 \times 100 \mu \mathrm{m}$ in the LSd and of $100 \times 300 \mu \mathrm{m}$ in the LSv and MS. The computer automatically counted all positive targets excluding noncellular irregularities in the background staining. This method, intermediate between stereological and exhaustive counting, also permits us to express results as the number of c-Fos-immunopositive cells per square millimeter for each region. At all stages the experimenter was blind to the experimental groupings.

\section{Data analysis}

Statistical analyses were performed using analysis of variance (ANOVA) (Statview, SAS Institute Inc.), followed by post hoc analyses (Tukey-Kramer) when appropriate. 


\section{Acknowledgments}

This study was supported by the Centre National de la Recherche Scientifique.

\section{References}

Anagnostaras, S.G., Gale, G.D., and Fanselow, M.S. 2001. Hippocampus and contextual fear conditioning: Recent controversies and advances. Hippocampus 11: 8-17.

Bannerman, D.M., Matthews, P., Deacon, R.M., and Rawlins, J.N. 2004 Medial septal lesions mimic effects of both selective dorsal and ventral hippocampal lesions. Behav. Neurosci. 118: 1033-1041.

Borhegyi, Z., Varga, V., Szilagyi, N., Fabo, D., and Freund, T.F. 2004. Phase segregation of medial septal GABAergic neurons during hippocampal theta activity. J. Neurosci. 24: 8470-8479.

Brady, J.V. and Nauta, W.J. 1953. Subcortical mechanisms in emotional behavior: Affective changes following septal forebrain lesions in the albino rat. J. Comp. Physiol. Psychol. 46: 339-346.

Calandreau, L., Desmedt, A., Decorte, L., and Jaffard, R. 2005. A different recruitment of the lateral and basolateral amygdala promotes contextual or elemental conditioned association in Pavlovian fear conditioning. Learn. Mem. 12: 383-388.

Calandreau, L., Trifilieff, P., Mons, N., Costes, L., Marien, M., Marighetto, A., Micheau, J., Jaffard, R., and Desmedt, A. 2006 Extracellular hippocampal acetylcholine level controls amygdala function and promotes adaptive conditioned emotional response. $J$. Neurosci. 26: 13556-13566.

Cho, Y.H., Friedman, E., and Silva, A.J. 1999. Ibotenate lesions of the hippocampus impair spatial learning but not contextual fear conditioning in mice. Behav. Brain Res. 98: 77-87.

Daumas, S., Halley, H., Francès, B., and Lassalle, J.M. 2005. Encoding, consolidation, and retrieval of contextual memory: Differential involvement of dorsal CA3 and CA1 hippocampal subregions. Learn. Mem. 12: 375-382.

Desmedt, A., Garcia, R., and Jaffard, R. 1998. Differential modulation of changes in hippocampal-septal synaptic excitability by the amygdala as a function of either elemental or contextual fear conditioning in mice. J. Neurosci. 18: 480-487.

Desmedt, A., Garcia, R., and Jaffard, R. 1999. Vasopressin in the lateral septum promotes elemental conditioning to the detriment of contextual fear conditioning in mice. Eur. J. Neurosci. 11: 3913-3921.

Desmedt, A., Garcia, R., and Jaffard, R. 2003a. An 8-day extensive elemental, but not contextual, fear conditioning potentiates hippocampal-lateral septal synaptic efficacy in mice. Synapse 49: $270-278$.

Desmedt, A., Hazvi, S., and Dudai, Y. 2003b. Differential pattern of cAMP response element-binding protein activation in the rat brain after conditioned aversion as a function of the associative process engaged: Taste versus context association. J. Neurosci. 23: 6102-6110.

Dragoi, G., Carpi, D., Recce, M., Csicsvari, J., and Buzsaki, G. 1999. Interactions between hippocampus and medial septum during sharp waves and theta oscillation in the behaving rat. J. Neurosci. 19: 6191-6199.

Fanselow, M.S. 1980. Conditional and unconditional components of post-shock freezing. Pavlov. J. Biol. Sci. 15: 177-182.

Feldon, J. and Gray, J.A. 1979. Effects of medial and lateral septal lesions on the partial reinforcement extinction effect at short inter-trial intervals. Q. J. Exp. Psychol. 31: 675-690.

Frankland, P.W., Cestari, V., Filipkowski, R.K., McDonald, R.J., and Silva, A.J. 1998. The dorsal hippocampus is essential for context discrimination but not for contextual conditioning. Behav. Neurosci. 112: 863-874.

Franklin, K.B.J. and Paxinos, G. 1997. The mouse brain in stereotaxic coordinates. Academic Press, New York.

Garcia, R. and Jaffard, R. 1996. Changes in synaptic excitability in the lateral septum associated with contextual and auditory fear conditioning in mice. Eur. J. Neurosci. 8: 809-815.

Gerlai, R. 1998. Contextual learning and cue association in fear conditioning in mice: A strain comparison and a lesion study. Behav. Brain Res. 95: 191-203.

Gray, J.A. 1982. Neuropsychological theory of anxiety: An investigation of the septal-hippocampal system. Cambridge University Press, Cambridge.

Gray, J.A. and McNaughton, N. 1983. Comparison between the behavioural effects of septal and hippocampal lesions: A review. Neurosci. Biobehav. Rev. 7: 119-188.

Gray, J.A. and McNaughton, N. 2000. The neuropsychology of anxiety: An enquiry into the function of the septo-hippocampal system, Vol. XVI. Oxford University Press, Oxford.
Hagan, J.J., Salamone, J.D., Simpson, J., Iversen, S.D., and Morris, R.G. 1988. Place navigation in rats is impaired by lesions of medial septum and diagonal band but not nucleus basalis magnocellularis. Behav. Brain Res. 27: 9-20.

Holland, P.C. and Bouton, M.E. 1999. Hippocampus and context in classical conditioning. Curr. Opin. Neurobiol. 9: 195-202.

Jakab, R.L. and Leranth, C. 1995. Septum. In The rat nervous system (ed. G. Paxinos), pp. 405-442. Academic Press, New York.

Kelsey, J.E. and Vargas, H. 1993. Medial septal lesions disrupt spatial, but not nonspatial, working memory in rats. Behav. Neurosci. 107: $565-574$

King, C., Recce, M., and O'Keefe, J. 1998. The rhythmicity of cells of the medial septum/diagonal band of Broca in the awake freely moving rat: Relationships with behaviour and hippocampal theta. Eur. J. Neurosci. 10: $464-477$.

Kirby, B.P. and Rawlins, J.N. 2003. The role of the septo-hippocampal cholinergic projection in T-maze rewarded alternation. Behav. Brain Res. 143: $41-48$.

Malpeli, J.G. and Schiller, P.H. 1979. A method of reversible inactivation of small regions of brain tissue. J. Neurosci. Methods 1: 143-151.

Maren, S., Aharonov, G., and Fanselow, M.S. 1997. Neurotoxic lesions of the dorsal hippocampus and Pavlovian fear conditioning in rats. Behav. Brain Res. 88: 261-274.

Martin, J.H. 1991. Autoradiographic estimation of the extent of reversible inactivation produced by microinjection of lidocaine and muscimol in the rat. Neurosci. Lett. 127: 160-164.

McAlonan, G.M., Dawson, G.R., Wilkinson, L.O., Robbins, T.W., and Everitt, B.J. 1995a. The effects of AMPA-induced lesions of the medial septum and vertical limb nucleus of the diagonal band of Broca on spatial delayed non-matching to sample and spatial learning in the water maze. Eur. J. Neurosci. 7: 1034-1049.

McAlonan, G.M., Wilkinson, L.S., Robbins, T.W., and Everitt, B.J. 1995b. The effects of AMPA-induced lesions of the septo-hippocampal cholinergic projection on aversive conditioning to explicit and contextual cues and spatial learning in the water maze. Eur. J. Neurosci. 7: 281-292.

Melia, K.R., Sananes, C.B., and Davis, M. 1992. Lesions of the central nucleus of the amygdala block the excitatory effects of septal ablation on the acoustic startle reflex. Physiol. Behav. 51: 175-180.

Menard, J. and Treit, D. 1996. Lateral and medial septal lesions reduce anxiety in the plus-maze and probe-burying tests. Physiol. Behav. 60: $845-853$.

Pesold, C. and Treit, D. 1992. Excitotoxic lesions of the septum produce anxiolytic effects in the elevated plus-maze and the shock-probe burying tests. Physiol. Behav. 52: 37-47.

Peterson, S.L. 1998. Drug microinjection in discrete brain regions. Kopf Carrier 50: 1-6.

Phillips, R.G. and LeDoux, J.E. 1992. Differential contribution of amygdala and hippocampus to cued and contextual fear conditioning. Behav. Neurosci. 106: 274-285.

Rawlins, J.N. and Olton, D.S. 1982. The septo-hippocampal system and cognitive mapping. Behav. Brain Res. 5: 331-358.

Rescorla, R.A. and Wagner, A.R. 1972. A theory of Pavlovian conditioning: Variations in the effectiveness of reinforcement and nonreinforcement. In Classical conditioning II: Current theory and research (eds. A.H. Black and W.F. Prokasy), pp. 65-99. Appleton-Century-Crofts, New York.

Richmond, M.A., Yee, B.K., Pouzet, B., Veenman, L., Rawlins, J.N., Feldon, J., and Bannerman, D.M. 1999. Dissociating context and space within the hippocampus: Effects of complete, dorsal, and ventral excitotoxic hippocampal lesions on conditioned freezing and spatial learning. Behav. Neurosci. 113: 1189-1203.

Selden, N.R., Everitt, B.J., Jarrard, L.E., and Robbins, T.W. 1991. Complementary roles for the amygdala and hippocampus in aversive conditioning to explicit and contextual cues. Neuroscience 42: 335-350.

Sheehan, T.P., Chambers, R.A., and Russell, D.S. 2004. Regulation of affect by the lateral septum: Implications for neuropsychiatry. Brain Res. Brain Res. Rev. 46: 71-117.

Sparks, P.D. and LeDoux, J.E. 1995. Septal lesions potentiate freezing behavior to contextual but not to phasic conditioned stimuli in rats. Behav. Neurosci. 109: 184-188.

Sparks, P.D. and LeDoux, J.E. 2000. The septal complex as seen through the context of fear. In The behavioral neuroscience of the septal region (ed. R. Numan), pp. 234-269. Springer-Verlag, New York.

Swanson, L.W. and Risold, P.Y. 2000. On the basic architecture of the septal region. In The behavioral neuroscience of the septal region (ed. R. Numan), pp. 1-14. Springer-Verlag, New York.

Tehovnick, E.J. and Sommer, M.A. 1997. Effective spread and timecourse of neural inactivation caused by lidocaine injection in monkey cerebral cortex. J. Neurosci. Methods 74: 17-26.

Thomas, E. 1988. Forebrain mechanism in the relief of fear: The role of

\section{Learning \& Memory


the lateral septum. Psychobiology 16: 36-44.

Thomas, E. and Yadin, E. 1980. Multiple-unit activity in the septum during Pavlovian aversive conditioning: Evidence for an inhibitory role of the septum. Exp. Neurol. 69: 50-60.

Thomas, E., Yadin, E., and Strickland, C.E. 1991. Septal unit activity during classical conditioning: A regional comparison. Brain Res. 547: 303-308.

Thomas, E., Strickland, C.E., Yadin, E., and Burock, D.A. 2005. Effects of chlordiazepoxide on single-unit activity in the septal region of the freely moving rat: Aversive vs. non-aversive contexts. Pharmacol. Biochem. Behav. 80: 151-159.
Trifilieff, P., Herry, C., Vanhoutte, P., Caboche, J., Desmedt, A., Riedel, G., Mons, N., and Micheau, J. 2006. Foreground contextual fear memory consolidation requires two independent phases of hippocampal ERK/CREB activation. Learn. Mem. 13: 349-358.

Yadin, E. and Thomas, E. 1981. Septal correlates of conditioned inhibition and excitation in rats. J. Comp. Physiol. Psychol. 95: $331-340$.

Received January 12, 2007; accepted in revised form April 24, 2007. 


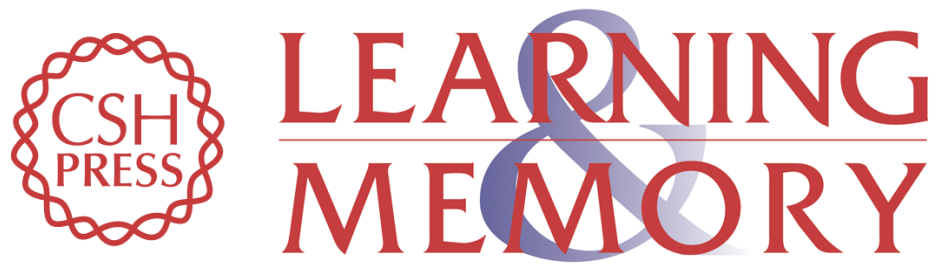

\section{Dissociated roles for the lateral and medial septum in elemental and contextual fear conditioning}

Ludovic Calandreau, Robert Jaffard and Aline Desmedt

Learn. Mem. 2007, 14:

Access the most recent version at doi:10.1101//m.531407

References This article cites 46 articles, 8 of which can be accessed free at:

http://learnmem.cshlp.org/content/14/6/422.full.html\#ref-list-1

License

Email Alerting Receive free email alerts when new articles cite this article - sign up in the box at the Service top right corner of the article or click here. 